\title{
Stimulus control of anticipatory responding in instrumental learning as revealed in serial learning tasks
}

\author{
E. J. CAPALDI and RONALD M. MILLER \\ Purdue University, West Lafayette, Indiana
}

\begin{abstract}
Items in a fixed series A-B-C can be anticipated employing either item information (e.g., Item A cues signal Item B) or position information (e.g., Position 2 cues signal Item B). Identical fixed series of rewarded and nonrewarded trials were employed in investigations reported by Capaldi and Miller (1988), Capaldi, Alptekin, Miller, and Birmingham (1997), and Burns, Dunkman, and Detloff (1999). These series were learned employing item cues according to Capaldi and colleagues and position cues according to Burns et al. (1999). Burns et al.'s (1999) conclusions were based on rats' behavior when shifted to novel series, which could reveal position learning but not item learning. Employing the acquisition conditions of Burns and co-workers but shifts to series that could reveal item learning, evidence for item learning was obtained in two experiments. The most reasonable interpretation of the above set of findings is that rats employ a compound of both types of cues to signal items. To our knowledge, no set of prior findings so strongly suggests a similar conclusion.
\end{abstract}

Examples of the numerous and diverse situations in which performance depends critically on learning the specific order in which items of a series (A-B-C-D-...) occur include phone numbers, days of the week, the alphabet, and choice points in multiple unit mazes. Typically, serial learning has been explained as a case of discrimination learning in which distinctive stimuli signal distinctive items. Two views of serial learning that have been rivals since the 1890s (see, e.g., Woodworth, 1938) disagree as to the origin or source of the distinctive stimuli that signal items. According to the item view, the stimuli arise from one or more prior items in the list (e.g., stimuli associated with Item A signal Item B). According to the position view, the stimuli arise from the position of the item in the list (e.g., stimuli associated with Position 2 in the list signal Item B). Some recent animal investigations support the item view, others the position view. It is important to realize, however, that evidence favoring item learning does not necessarily rule out position learning and vice versa, a point often overlooked.

Capaldi and Miller (1988) trained rats in a gray runway under two different series, XNY and ZNN, and obtained evidence for item learning. $\mathrm{X}, \mathrm{Y}$, and $\mathrm{Z}$ were discriminably different food items, and $\mathrm{N}$ was a nonreward. Items of a series were separated by a $15-30$-sec interval, a series being separated by about a 10-15-min interval. There was a single presentation of each series each day, the two series being presented in irregular order over days. Capaldi and Miller reported that with training, rats devel-

Address correspondence to E. J. Capaldi, Department of Psychological Sciences, 1364 PSYC, Purdue University, West Lafayette, IN 47907-1364 (e-mail: julie@ psych.purdue.edu). oped appropriate serial responding, defined as running fast to X, Y, and Z and slow to all Ns. More specifically, running was equally rapid on Trial 1 of the two series, but on Trials 2 and 3 running was faster in the XNY series than in the ZNN series.

Running faster on Trial 2 of the XNY series than on Trial 2 of the XNN series, an $\mathrm{N}$ trial in both series, indicated that, on Trial 2, the rats were anticipating the Trial 3 event (i.e., Y or N). This phenomenon was called interevent anticipation (see also, Capaldi, 1992). Under the $\mathrm{XNY}$ and ZNN series, Position 3 cues were associated with both Y (XNY series) and N (ZNN series), so position cues cannot explain either interevent anticipation on Trial 2 or discriminative responding on Trial 3. But item learning can by assuming that, on any given trial, animals may remember each of the reward events that occurred on one or more prior trials (see, e.g., Capaldi, 1992, 1994; Capaldi \& Birmingham, 1998). Specifically, in the XNY series, the rats remembered $\mathrm{X}$ and $\mathrm{X}+\mathrm{N}$ on Trials 2 and 3, respectively, whereas, in the $\mathrm{ZNN}$ series, the rats remembered $\mathrm{Z}$ and $\mathrm{Z}+\mathrm{N}$, respectively on Trials 2 and 3 . Thus, the memory of $X$ became a signal for reward on Trial 3, whereas $\mathrm{Z}$ signaled nonreward on Trial 3 as well as on Trial 2. Accordingly, running will be faster when $X$ is remembered compared with when $\mathrm{Z}$ is remembered, which explains interevent anticipation and serial responding from an item standpoint.

Additional findings consistent with an item interpretation were reported by Capaldi, Alptekin, Miller, and Birmingham (1997). In Experiment 1 of that report, a group with item relevant but not position relevant acquired serial responding more rapidly than a group with position but not item relevant. The item relevant group received, each day, in a gray runway, three different series 
in irregular order, SN, PSN, and PPSN. The position relevant group received, each day, the series PPN, PSN, SSN, and SPN. Over days the two groups received the same number of $\mathrm{P}, \mathrm{S}$, and $\mathrm{N}$ trials ( $\mathrm{P}$ and $\mathrm{S}$ were food items), the same number of transitions from food trials to $\mathrm{N}$ trials, and the mean position of $\mathrm{N}$ in the series was three for both groups. In the item relevant group, the immediately prior item (S) was a reliable signal for $\mathrm{N}$, but this was not the case in the position relevant group. ${ }^{1}$ Prior to a subsequent shift phase, the item relevant group, but not the position relevant group, acquired the tendency to run slow on $\mathrm{N}$ trials, whereas both groups ran rapidly on $\mathrm{P}$ and $\mathrm{S}$ trials. This finding supports the item view. The shift findings produced further support for the item view: On being shifted to a series in which $\mathrm{N}$ was in a novel position (PPPSN), slow running to $\mathrm{N}$ occurred immediately in the item relevant group but not in the position relevant group. Too, running was rapid on Position 4 of the PPPSN series in the item relevant group. Position 4 was consistently nonrewarded and was associated with slow running under the PPSN series. In Experiment 2 of Capaldi et al. (1997), rats trained PSN (item cues and position cues relevant) did not acquire serial responding any more rapidly than rats trained SN, PSN, and PPSN (item cues but not position cues relevant), suggesting that position cues were ineffective.

Recently very provocative and interesting findings supporting a position view were reported by Burns, Dunkman, and Detloff (1999) and by Burns, Kinney, and Criddle (2000). In the Burns et al. (1999) investigations, rats received the $\mathrm{XNY}$ series in one brightness alternative (e.g., black) and the ZNN series in another brightness alternative (e.g., white), with the series occurring irregularly. Under this training, interevent anticipation and serial responding took the same form as reported by Capaldi and Miller (1988), who employed the XNY and ZNN series in a gray runway. Following the development of serial responding in each alternative in preshift, the rats were shifted to either NNN in both black and white, or $\mathrm{ZNY}$ in black and XNN in white, or ZNN in black and $\mathrm{XNY}$ in white. In each of these shifts, rats responded as in preshift (i.e., fast, slow, fast over the three successive trials in black, and fast, slow, slow over the three successive trials in white). These postshift results indicated that serial responding in preshift was controlled by position cues since relevant position cues but not item cues were common to both preshift and postshift. Similarly, Burns et al. (2000) reported that in a gray runway, two groups, one trained PSN, the other PPN, PSN, SSN, and SPN, having acquired serial responding in preshift, continued to manifest it when shifted to NNN, supporting position learning. Although the above cited investigations of Burns and his colleagues support the position view, they do not rule out the possibility that item learning had also occurred, a matter of concern in the present experiments.

A comparison of the previously mentioned findings reported by Capaldi and colleagues (e.g., Capaldi, 1994; Neath \& Capaldi, 1996) with those reported by Burns et al.
(1999) lends support to the views suggested by some that in serial learning situations animals may employ either item cues or position cues. However, when and under what conditions animals employ either item cues or position cues, or both, despite over 100 years of investigation, is still a matter of speculation. As one example, Burns et al. (1999) suggested, in effect, that animals are primarily disposed to employing position cues, but will employ item cues if they provide information about items that is not provided by position cues. For example, under the XNY and ZNN series (gray runway) in which Trial 3 position cues do not validly signal whether $Y$ or $\mathrm{N}$ will occur, Burns et al. (1999) suggested that item information from the Trial 1 events, $\mathrm{X}$ or $\mathrm{Z}$, was combined with Trial 3 position information so as to correctly signal the Trial 3 events. But, under XNY and ZNN training in which brightness cues validly signal which series will occur, items can be and are anticipated by employing only position cues according to Burns et al. (1999). On the other hand, Capaldi et al. (1997) suggested (see also Capaldi, 1994) that when item cues and position cues are simultaneous valid predictors of items, as they usually are, mutual overshadowing occurs, with greater control being exercised by the more salient of the two cues, usually the item cue (see also Capaldi, Verry, Nawrocki, $\&$ Miller, 1984). Even though the overshadowing view suggested here may be radical in the area of serial learning (e.g., neither overshadowing nor blocking is even mentioned in human serial learning), it is, in other respects, rather conventional. It merely suggests that item cues and position cues, in common with other cues, may overshadow and block each other.

The two experiments reported here attempt to determine whether no item learning occurs (Burns et al., 1999) or whether some item learning occurs (e.g., Capaldi et al., 1997) when both item cues and position cues are simultaneously valid, as when XNY and ZNN series are given in black and white runways, respectively (Experiment 1), or as when PSN training is given in a gray runway (Experiment 2). In each experiment, there was a preshift phase, in which both position cues and item cues validly, predicted all items, and a postshift phase, in which a novel test for item learning occurred. In the preshift phase of Experiment 1, the XNY and ZNN series occurred in different brightness alternatives (e.g., XNY in black and $\mathrm{ZNN}$ in white). In postshift, there were two groups, Group Same and Group Different, both of which were trained in a gray runway. In postshift, the series occurred as it did in preshift for Group Same (i.e., XNY and ZNN). However, for Group Different, the series was rearranged as follows, ZNY and XNN. In preshift, the black and white runways indicated whether Position 3 was to terminate in $\mathrm{Y}$ or in N. In postshift, however, the gray runway failed to indicate whether Position 3 terminated in $\mathrm{Y}$ or in $\mathrm{N}$, but, of course, Trial 1 items were able to supply that information in postshift as they did in preshift. If no item learning occurred in preshift, it should not matter in postshift whether the Trial 1 item signals the same Trial 3 
item, as in preshift (Group Same), or different Trial 3 items, as in preshift (Group Different), and so, in postshift, Groups Same and Different should not differ either on Trial 2 or on Trial 3. But, if some item learning occurs in preshift, interevent anticipation and serial responding should be better in postshift in Group Same than in Group Different.

In Experiment 2, preshift and postshift occurred in a gray runway. In Experiment 2 in preshift, both item and position cues were relevant in Group IP (Group IP was trained PSN), but only item cues were relevant in Group I (Group I was trained SN, PSN, and PPSN). In a novel shift condition, Group IP was given the same training as Group I (i.e., SN, PSN, PPSN). In postshift, the N event occurred in a new position for Group IP in two of the three postshift series, the SN series and the PPSN series. If, in preshift, as the view of Burns et al. (1999) suggests, Group IP learned only about position, it should not, in postshift, manifest serial responding under either the SN series or the PPSN series, much less respond as appropriately to these series as Group I. However, to the extent that Group IP learns in preshift that item $\mathrm{S}$ is a valid predictor of $\mathrm{N}$, it should, in postshift, manifest serial responding in connection with the SN and PPSN series.

\section{EXPERIMENT 1}

In preshift, as indicated, the rats received the XNY and ZNN series in different brightness alternatives (e.g., $\mathrm{XNY}$ in black and ZNN in white). In postshift, the rats were shifted to a gray runway and were trained either XNY and ZNN (Group Same) or ZNY and XNN (Group Different).

\section{Method}

Subjects. The subjects were 8 male albino rats about 80 days old on arrival at the laboratory, purchased from the Holtzman Co., Indianapolis.

Apparatus. The apparatus consisted of three side-by-side runways identical except for brightness. One was black, one was white, and one was gray. The startboxes, also black, white, or gray, were $21.61 \mathrm{~cm}$ long. The goal boxes were $33.95 \mathrm{~cm}$ long. Each alley was $8.5 \mathrm{~cm}$ wide and a total of $190.8 \mathrm{~cm}$ long. Each alley was enclosed by a wire mesh top on a hinged frame. Raising the startbox door started a timer in a computer (IBM PC XT) that was stopped when the rat broke a photobeam located $158.13 \mathrm{~cm}$ beyond the startbox door. Food rewards, either six .045-gm Noyes pellets (X) or a Kellogg's corn pop (Y), or a Kellogg's sugar smack (Z), was placed in the $3.8 \times 3.8 \times 0.95 \mathrm{~cm}$ goal cup at the end of the runway. The pellets were not visible before the photobeam was broken. A brass door confined the rat to the goal box.

Pretraining. The rats were caged individually and given food and water ad lib for 14 days after arriving at the laboratory. On Day 15, pretraining began. On Day 1 of pretraining, food deprivation began, consisting of $18 \mathrm{~g}$ per day of LAB Rodent Diet 5001. On Days 1-7 of pretraining, the rats were handled. On Days 6-7 of pretraining, the rats received, respectively, six pellets, a pop, and a smack in the home cage (the same three food items in the black alley and the same three food items in the white alley). No training was given in the gray alley.

Experimental Training. Experimental training began on Day 23 and consisted of two phases, preshift and postshift. In preshift, the series was presented in one of four different arrangements, 2 rats in each. These were: XNY and ZNN in black and white, respectively; ZNY and XNN in black and white, respectively; XNY and ZNN in white and black, respectively; and ZNY and $\mathrm{XNN}$ in white and black, respectively. In postshift, 1 rat from each of the four subgroups received the same series as in preshift in a gray runway (Group Same). The other rat from the subgroup received the reverse of the Trial 1 events (Group Different). To be specific, the rats that were trained XNY and ZNN in preshift, were, in postshift, trained either XNY and ZNN (Group Same) or ZNY and XNN (Group Different).

Importantly, in order to simplify exposition, henceforth all rats are said to have been trained in preshift, $\mathrm{XNY}$ and $\mathrm{ZNN}$ in black and white runways, respectively.

The interval elapsing between trials of a series was about $30 \mathrm{sec}$. Each rat received its first scheduled series before any rat received its second scheduled series. This produced an interseries interval of about $20 \mathrm{~min}$. The series terminating in $\mathrm{Y}$ is 1 , and the series terminating in $\mathrm{N}$ is 2 . The order of presenting the series over repeated 4-day blocks was 1212 (Day 1), 2211 (Day 2), 1122 (Day 3), and 2121 (Day 4). However, to present the first four series (1212) required the first 3 days of experimental training-Day 1 (1), Day 2 (2), Day 3 (12). Counting Days 1-3 as Day 1, there were 22 days of preshift.

There were 10 days of postshift. Experimental conditions in postshift were the same as in preshift, except that the rats were trained in the gray runway, either XNY and ZNN, or ZNY and XNN.

\section{Results}

Differences are significant at $p<.05$.

Preshift. Figure 1 shows running speed on each of the trials of the XNY and ZNN series on each of the 22 days of preshift. Henceforth, the series terminating in $\mathrm{Y}$ is called the plus series, and the series terminated in $\mathrm{N}$, the minus series. On Trial 1, running speed differed little between the two series. On Trials 2 and 3, however, running was faster in the plus series than in the minus series. Thus, interevent anticipation occurred (Trial 2), and on Trial 3, the rats ran faster to reward than to nonreward. These findings are consistent with those obtained in Burns et al. (1999), in which rats were trained as here in preshift, and with those obtained in Capaldi and Miller(1988), in which rats were trained $\mathrm{XNY}$ and $\mathrm{ZNN}$ in a gray runway.

An analysis of variance (ANOVA) was applied to the data shown in Figure 1. In the analysis, the same and different groups were included as dummy variables. Analysis revealed that neither the main effect nor any interaction associated with same versus different was significant. Of major interest for the present purposes, analysis revealed that significant differences were associated with series $[F(1,24)=113.24]$, with trials $[F(2,48)=251.89]$, with the series $\times$ trials interaction $[F(2,48)=80.60]$, and with the series $\times$ days $\times$ trials interaction $[F(42,1008)=3.65]$. Subsequent Newman-Keuls tests indicated that differences due to series, though not significant on Trial 1, were significant on Trials 2 and 3.

\section{Postshift}

Figure 2 shows running speed in postshift for Groups Same and Different in the plus and minus series on Trial 2 in blocks of 2 days. Figure 3 shows the same data for Trial 3. A separate ANOVA was applied to each trial in 


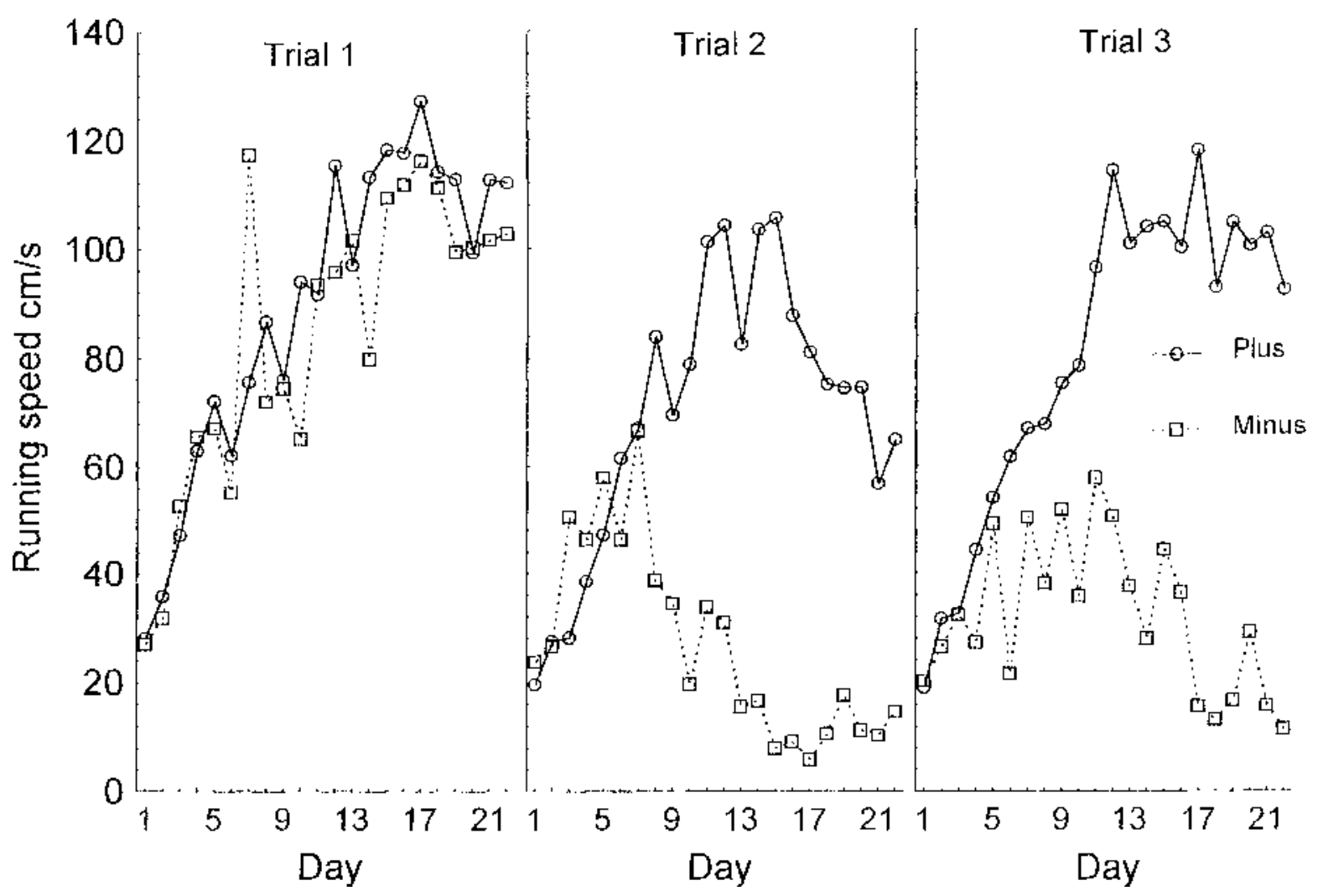

Figure 1. Running speed on each of the trials of the XNY and ZNN series on each of the 22 days of preshift. The series terminating in $\mathrm{Y}$ is called the plus series; the series terminating in $\mathrm{N}$ is called the minus series.

postshift. In postshift, as in preshift, no difference was significant on Trial 1 (data not shown). On Block 5 of postshift, for example, running speeds on Trial 1 in the plus versus minus trials were, respectively, 118.3 and 118.4 $\mathrm{cm} / \mathrm{sec}$ for Group Same and 117.2 and $122.2 \mathrm{~cm} / \mathrm{sec}$ for Group Different.

On Trial 2 on each block of postshift trials, Group Same ran faster in the plus series than in the minus series, whereas Group Different ran faster in the minus series than in the plus series. In short, Group Same and Group Different ran faster on Trial 2 when $\mathrm{X}$ rather than $\mathrm{Z}$ occurred on Trial 1, in both postshift and preshift. This produced, in postshift, appropriate interevent anticipation for Group Same but not for Group Different. An ANOVA indicated that, on Trial 2, although the difference associated with groups was not significant, the groups $\times$ series difference was $[F(1,56)=4.67]$.

On Trial 3, both groups ran slightly faster in the plus series than in the minus series on Block 1. In subsequent blocks, Group Different ran about equally rapidly in the plus and minus series. Although this was true for Group Same in Block 2, on the three subsequent blocks, Group Same ran faster in the plus series than in the minus series. An ANOVA revealed that, on Trial 3, all main effects (groups, series, blocks) and all interactions were sig- nificant [smallest $F=(4,220)=2.83$ for the series $\times$ blocks interaction].

\section{Discussion}

Burns et al. (1999), as previously mentioned, reported shift findings indicating that rats employed position cues when trained XNY and ZNN in black and white runways, respectively. Burns et al. (1999) said of those shift findings, "In this procedure, item memories of the particular reward outcomes did not control the discriminations established during the initial training phase" (p. 275). This conclusion is shown to be in error by the present finding. Two findings obtained in postshift indicated that, in preshift, under XNY and ZNN in black and white runways, respectively, responding was controlled by item memories. First, in postshift, from the outset of training on Trial 2, responding in both Group Same and in Group Different was consistent with the view that the expectancy of reward was greater following $X$ on Trial 1 than following $\mathrm{Z}$ on Trial 1 (i.e., in both groups in postshift, running was faster on Trial 2 following $X$ rather than $Z$ on Trial 1). In postshift, this produced appropriate interevent anticipation in Group Same and what could be called "inappropriate" interevent anticipation in Group Different. Second, on Trial 3 of postshift, Group Same but not 


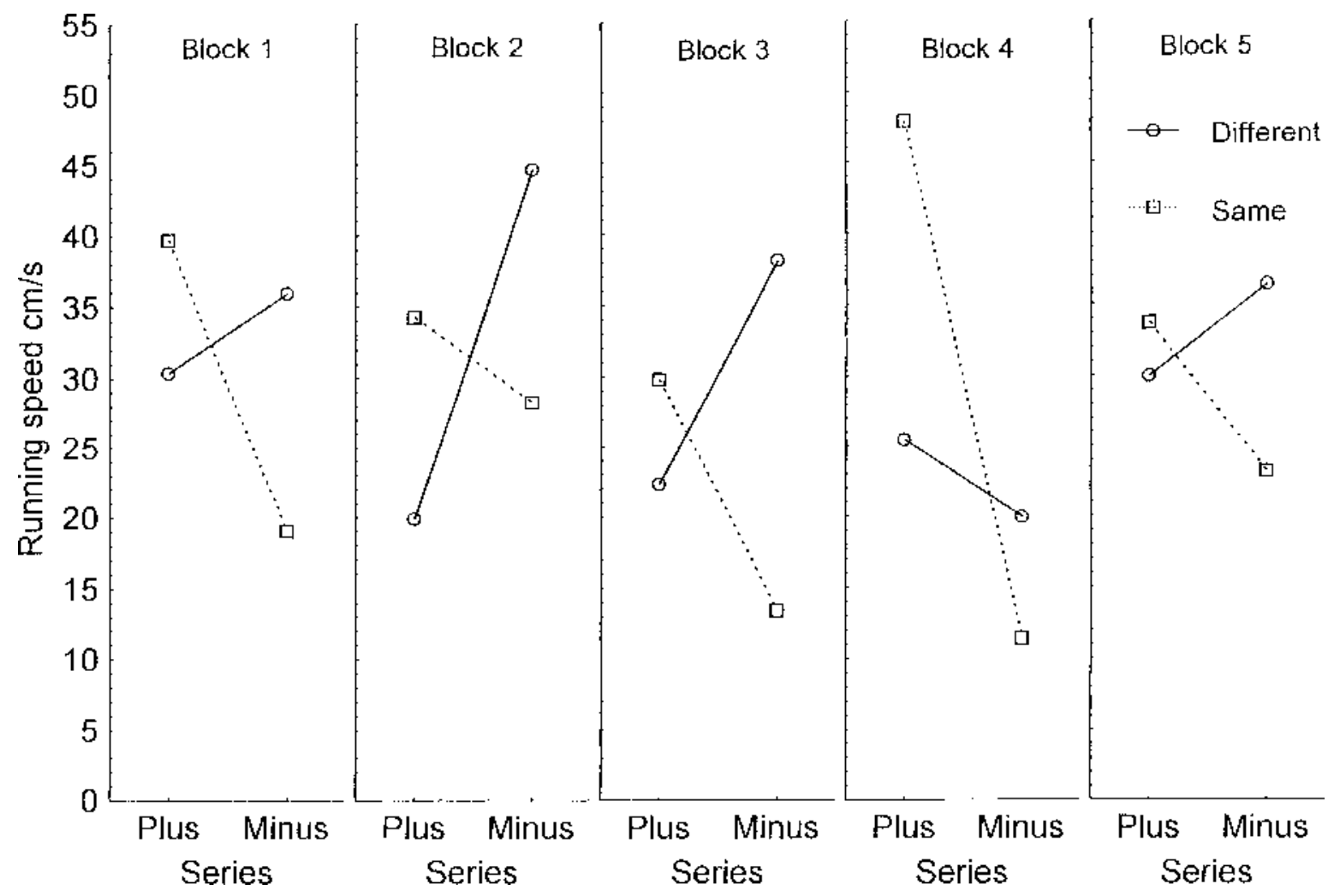

Figure 2. Running speed in postshift for Groups Same and Different in the plus and minus series on Trial 2.

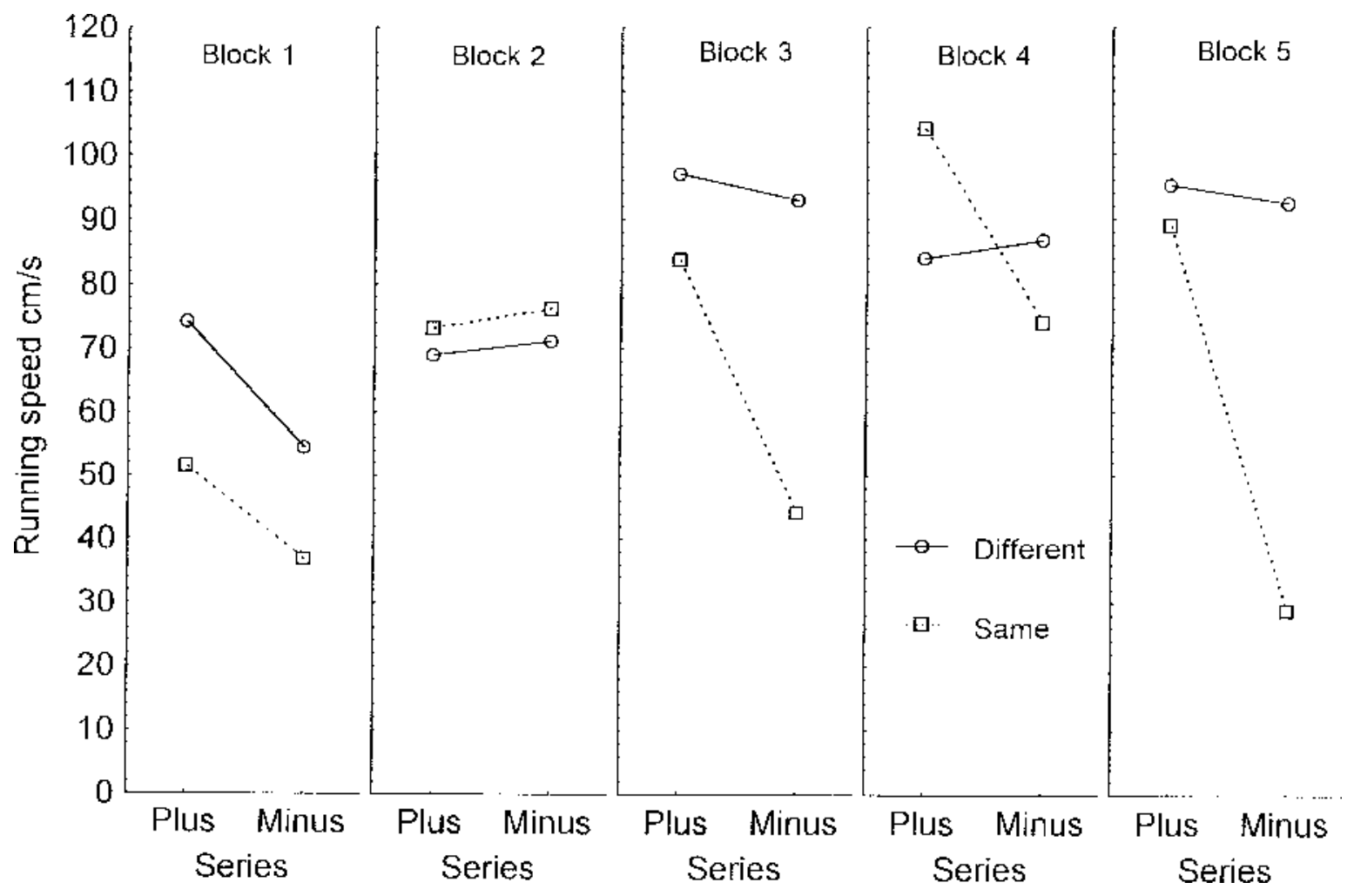

Figure 3. Running speed in postshift for Groups Same and Different in the plus and minus series on Trial 3. 
Group Different acquired the tendency to run faster in the plus series than in the minus series. But such discriminative responding did not appear in Group Same until the third block of postshift trials. The most reasonable interpretation of the preshift findings reported here and by Burns et al. (1999) is that items were signaled in both series, employing item cues and position cues in compound.

Why responding in postshift, which clearly reflected control by item memories, appeared much sooner on Trial 2 than on Trial 3 in both groups is a matter of conjecture. This finding could reflect a greater control over responding by item memories on Trial 2 than on Trial 3 . This may be because it is easier to discriminate between the memories of $X$ versus $Z$ (Trial 2) than between the memories $X+N$ versus $Z+N$ (Trial 3). Too, it may be related to the fact that, in preshift, Trial 2 was nonrewarded in both brightness alternatives, whereas Trial 3 was rewarded in one brightness alternative and nonrewarded in the other. If, as this reasoning suggests, in preshift, brightness was a more critical factor in signaling the Trial 3 item than the Trial 2 item, then a shift to a common brightness in postshift would be expected to disrupt responding more greatly on Trial 3 than on Trial 2 . According to this view, when rats are trained $\mathrm{XNY}$ and $\mathrm{ZNN}$ in black and white runways, respectively, the stimuli $X+N+$ black signal $Y$ on Trial 3, whereas the stimuli $Z+N+$ white signal $\mathrm{N}$ on Trial 3. In postshift, when XNY and ZNN occurred in the gray runway, the relevant brightness cues were removed from each of the Trial 3 stimulus compounds (i.e., $\mathrm{X}+\mathrm{N}+$ gray vs. $\mathrm{Z}+\mathrm{N}+$ gray) and so produced some disruption of responding on that trial.

Granting that rats employ both item cues and position cues to signal items when trained XNY and ZNN in black and white runways, respectively, the question arises: What is the role of each of these stimuli in producing interevent anticipation? According to Burns et al. (1999), under such training, interevent anticipation occurs because of generalization between position cues. That is, under such training, according to Burns et al. (1999), Position 3 cues would supply a stronger generalized approach tendency to Position 2 cues in the XNY series than in the ZNN series. This is because Position 3 cues were rewarded in the former series but not the latter series. The shift findings of Experiment 1 here clearly indicate that interevent anticipation was controlled by item memories, both when the XNY and ZNN series occurred either in black or white runways, respectively, and in a single gray runway. Given the findings obtained in Experiment 1, it is clear that there is as yet no direct evidence that, when XNY and ZNN occur in black and white runways, respectively, interevent anticipation is controlled by position cues. That hypothesis, given the present findings, can only be regarded as unsupported at present.

It is possible to quarrel with the conclusion suggested here that, when XNY and ZNN occur in black and white runways, respectively, both item learning and position learning occur. Recognize, first of all, that although Experiment 1 here and the experiments of Burns et al. (1999) are highly similar, they are not identical. Therefore, it could be argued that small differences between the two are such as to favor item learning in the present experiment and position learning in Burns et al. (1999). This suggestion seems highly unlikely, inasmuch as rather gross brightness differences between the XNY and ZNN series in a single gray runway or in black and white runways, respectively, nevertheless produced, as indicated, highly similar acquisition (preshift) findings in the two situations (compare Capaldi \& Miller, 1988, with Burns et al., 1999). It appears that acquisition findings obtained by employing the XNY and ZNN series are rather robust, being unaffected by even large difference in brightness conditions. On the basis of these prior findings, it seems unlikely that rats trained XNY and ZNN in black and white runways, respectively, learned different things in the present experiment and in Burns et al. (1999).

\section{EXPERIMENT 2}

Experiment 2 was conducted in four phases. In Phase 1, the rats were trained in a gray runway either PSN (Group IP, both item and position relevant) or SN, PSN, and PPSN (Group I, only item relevant). In Phase 2, both groups were trained PSN. The intention in Phase 2 was merely to determine whether training under the PSN alone (Group IP) would lead to better performance under that series than under SN, PSN, and PPSN (Group I). In Phase 3, both groups were trained as in Phase 1. In Phase 4, both groups were trained SN, PSN, and PPSN. Phase 4 was the most critical phase. In Phase 4, it was of interest to determine how well Group IP would perform under two novel series, SN and PPSN. If, under PSN training, Group IP learned only about position and not about item, it should not correctly anticipate the $\mathrm{N}$ item in either the $\mathrm{SN}$ series or the PPSN series. However, depending on the extent to which Group IP learns under PSN training that item S signals item $\mathrm{N}$, running should be slow to $\mathrm{N}$ in both the SN series and the PPSN series. Since Position 2 was consistently rewarded under PSN training, Group IP might experience more difficulty running slowly on the $\mathrm{N}$ trial of the SN series than on the $\mathrm{N}$ trial of the PPSN series. Under the PPSN series, the $\mathrm{N}$ trial occurs in a novel position (i.e., 4), which had not been rewarded previously in Group IP, unlike Position 2, which had been consistently rewarded prior to Phase 4.

\section{Method}

Subjects. The subjects were 8 rats of the same description as those employed in Experiment 1.

Apparatus. The same apparatus as that in Experiment 1 was employed in Experiment 2, using only the gray runway.

Pretraining. Pretraining was the same as in Experiment 1 except that the rats received $.045 \mathrm{~g}$ plain and sucrose pellets in the home cage, and three plain and three sucrose pellets in the gray runway.

Experimental training. The experiment was conducted in four phases. Series were constructed employing six plain pellets $(\mathrm{P})$, six sucrose pellets (S), and nonreward (N). In Phase 1, 2 rats in Group IP (for item and position relevant) received, each day, the series PSN, and 2 rats received the series SPN. Two rats in Group I (for item relevant) received, each day, the series SN, PSN, and PPSN, and 2 rats received, each day, the series PN, SPN, and SSPN. To simplify mat- 
ters, the rats in Group IP are said to have received the PSN series, and the rats in Group I are said to have received the SN, PSN, and PPSN series. Since Group IP received three presentations daily of the three trial series, it can be seen that, each day, all animals received nine total trials (three $\mathrm{S}$ trials, three $\mathrm{P}$ trials, and three $\mathrm{N}$ trials). Importantly, each group received, each day, three transitions from a rewarded trial to a nonrewarded trial.

Trials and series were presented in Experiment 2 as they were in Experiment 1. Group I received its two-, three-, and four-trial series in 6-day blocks as follows: 2, 3, 4 (Day 1); 4, 2, 3 (Day 2); 3, 4, 2 (Day 3); 2, 4, 3 (Day 4); 4, 3, 2 (Day 5); 3, 2, 4 (Day 6). However, to present the first three series, Group IP received one series on Day 1 and two series on Day 2. Group I received its two-trial series on Day 1 and its three- and four-trial series on Day 2. Counting Days 1 and 2 as a single day, there were 30 days of training in Phase 1 . Except for the changes noted below, the rats were trained in subsequent phases the same as in Phase 1.

Phase 2. On Days 31 and 32 of experimental training, all rats received the three-trial series PSN.

Phase 3. On Day 33 of experimental training, all rats were trained as they were in Phase 1.

Phase 4. On Day 34 of experimental training, all rats received a single presentation of each of three series, the two-trial, three-trial, and four-trial series.

\section{Results}

Differences are significant at $p<.05$.

In order to compare the two groups on a common basis, we utilized running speeds on the terminal nonrewarded trial and on the rewarded trial that immediately preceded it in each of the series of both groups. The speeds for each group are shown for each day of Phase 1 in Figure 4. The left panel shows speeds on rewarded trials; the right panel shows speeds on nonrewarded trials. Two notable trends can be seen in Figure 4. First, with training, both groups came to run more slowly to nonreward than to reward. Second, Group I ran more rapidly than Group IP. The tendency for Group I to run faster than Group IP was apparent in all phases of Experiment 2, but this difference did not in any comparison reach statistical significance. An ANOVA applied to the data shown in Figure 4 revealed that significant differences were associated with trials $[F(1,22)=38.71]$, days $[F(29,638)=15.71]$, and trials $\times$ days $[F(29,638)=8.43]$. However, no main effect or interaction associated with group was significant.

Figure 5 shows mean running speed in Phase 2 for Groups I and IP on each of the three trials of the PSN series on both of the 2 days of training. Both groups ran faster on each of the rewarded trials than on the nonrewarded trial. An ANOVA applied to the data shown in Figure 4 indicted that significant differences were associated with trials $[F(2,42)=42.34]$, with no other differences being significant.

In Phase 3, the groups were trained as in Phase 1, and performance data from Phase 3 were much like those presented in Figure 4. Accordingly, Phase 3 data are not shown. In Phase 3, as in Phase 1, the only significant difference was that associated with trials $[F(1,22)=38.37]$.

Figure 6 shows running speeds for both of the groups on each of the series received on Day 1 of Phase 4, the SN series (left panel), the PSN series (center panel), and the PPSN series (right panel). Although Group I ran faster overall than Group IP in each of the series, it appears that serial responding, fast running on rewarded trials and slow running on nonrewarded trials, were about the same for

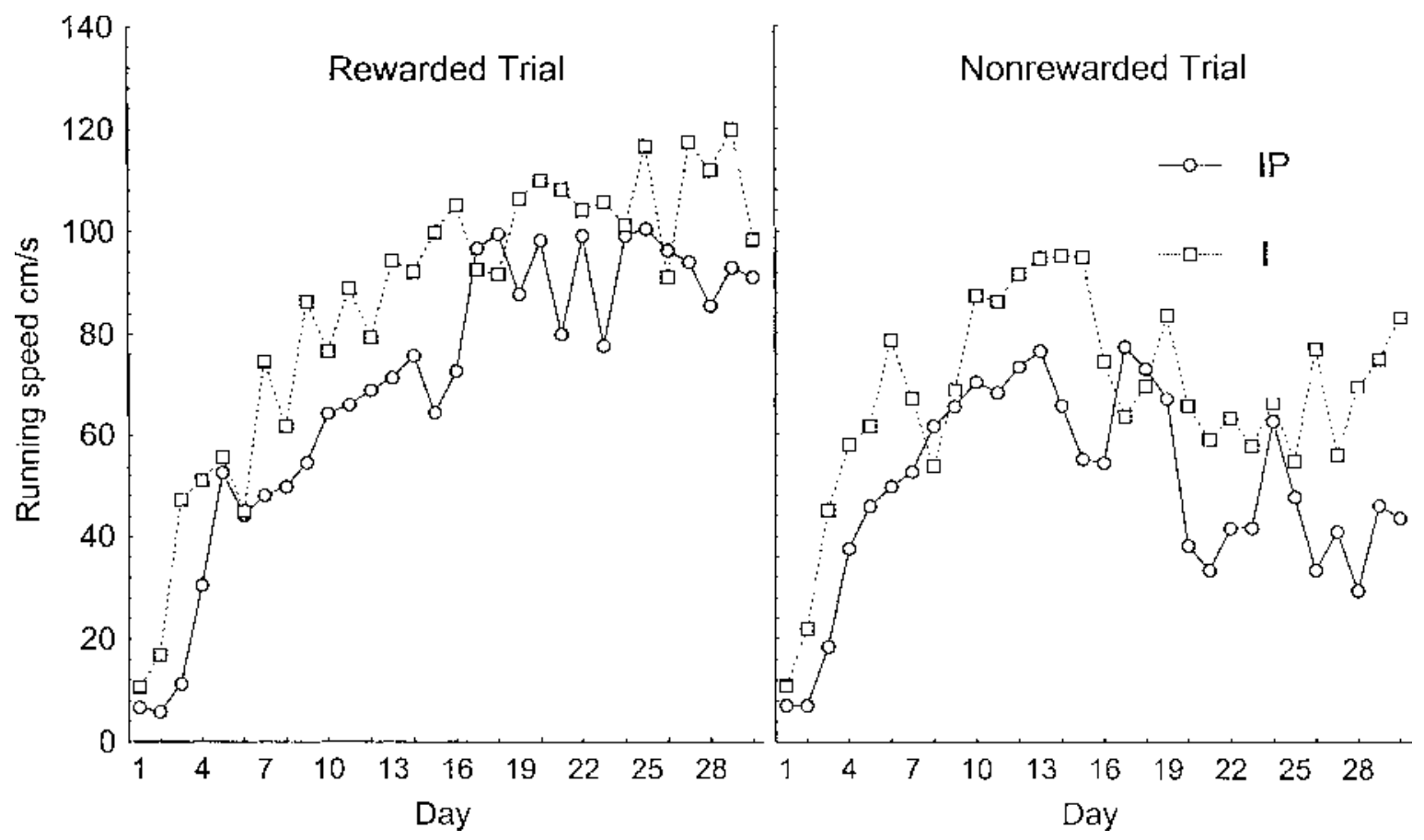

Figure 4. Running speed in Phase 1 for Groups IP and I on each of the 30 days of training on the rewarded trial that preceded the terminal nonrewarded trial in each series. 


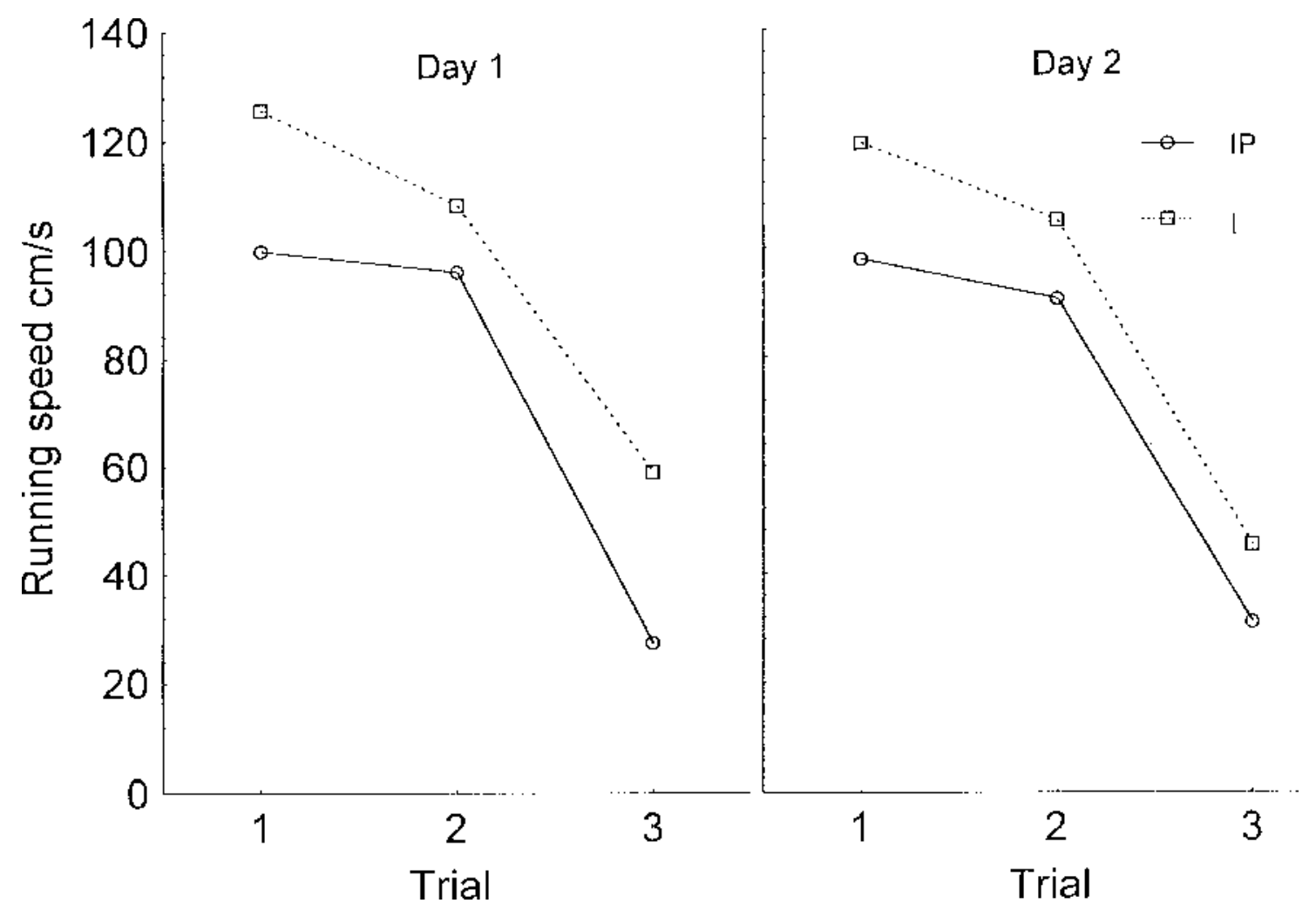

Figure 5. Running speed in Phase 2 for Groups IP and I on each of the trials of the PSN series on each of the 2 days of training.

both groups. An ANOVA applied to each series bears this out. In each series, the only significant difference was that associated with trials (i.e., no differences associated with group were significant). The difference associated with trials was significant in the SN series $[F(1,6)=37.09]$, in the PSN series $[F(2,12)=11.03]$, and in the PPSN series $[F(3,18)=8.24]$.

\section{Discussion}

Capaldi et al. (1997) reported that a group trained in a gray runway in such a way that item cues but not position cues validly signaled items in preshift (SN, PSN, and PPSN training) developed serial responding in preshift that was maintained in postshift when $\mathrm{N}$ occurred in a novel position (PPPN training). Burns et al. (2000) reported that a group for which both item cues and position cues validly signaled items in a single runway (PSN training) developed serial responding in preshift that was maintained when, in postshift, item cues were not available (NNN training). In the present experiment, the group trained PSN developed serial responding in preshift that was maintained in postshift when $\mathrm{N}$ occurred in a novel position, either SN training or PPSN training, indicating that the rats employed item cues in preshift. Note that, if rats trained PSN were learning to run slowly, following two rewarded trials, rather than employing items as a signal for N, they would not have run slowly on Trial 2 of the SN series. Thus, given previous findings, it appears that under PSN training, rats anticipate items by employing both position cues (Burns et al., 2000) and item cues (Capaldi et al., 1997; present Experiment 2). This conclusion is consistent with that offered in Experiment 1 in indicating that when both position cues and item cues are valid, rats can employ both sorts of cues to anticipate items.

In addition to Group IP, Experiment 2 employed, in Phase 1, a group in which item cues but not position cues validly signaled items (Group I). In Phase 2, both groups performed equally well when shifted to PSN. This finding seems to indicate that SN and PPSN training contribute as much to proper serial responding under the PSN series as direct training does under that series, a finding more consistent with item learning than with position learning. In Phase 4, Groups IP and I performed about equally well when trained SN, PSN, and PPSN. The Phase 4 results indicate that, in Group IP, contrary to the suggestion of Burns et al. (1999), valid position cues had a negligible effect (if any) in reducing item learning, a matter considered at greater length in the General Discussion section. In Phase 4, Group IP received an N trial in a novel position (PPSN series, Position 4) and in a position (Position 2, SN series) that had been rewarded a great number of times in each of the first three phases. The fact that Group IP ran slowly to nonreward on the very 


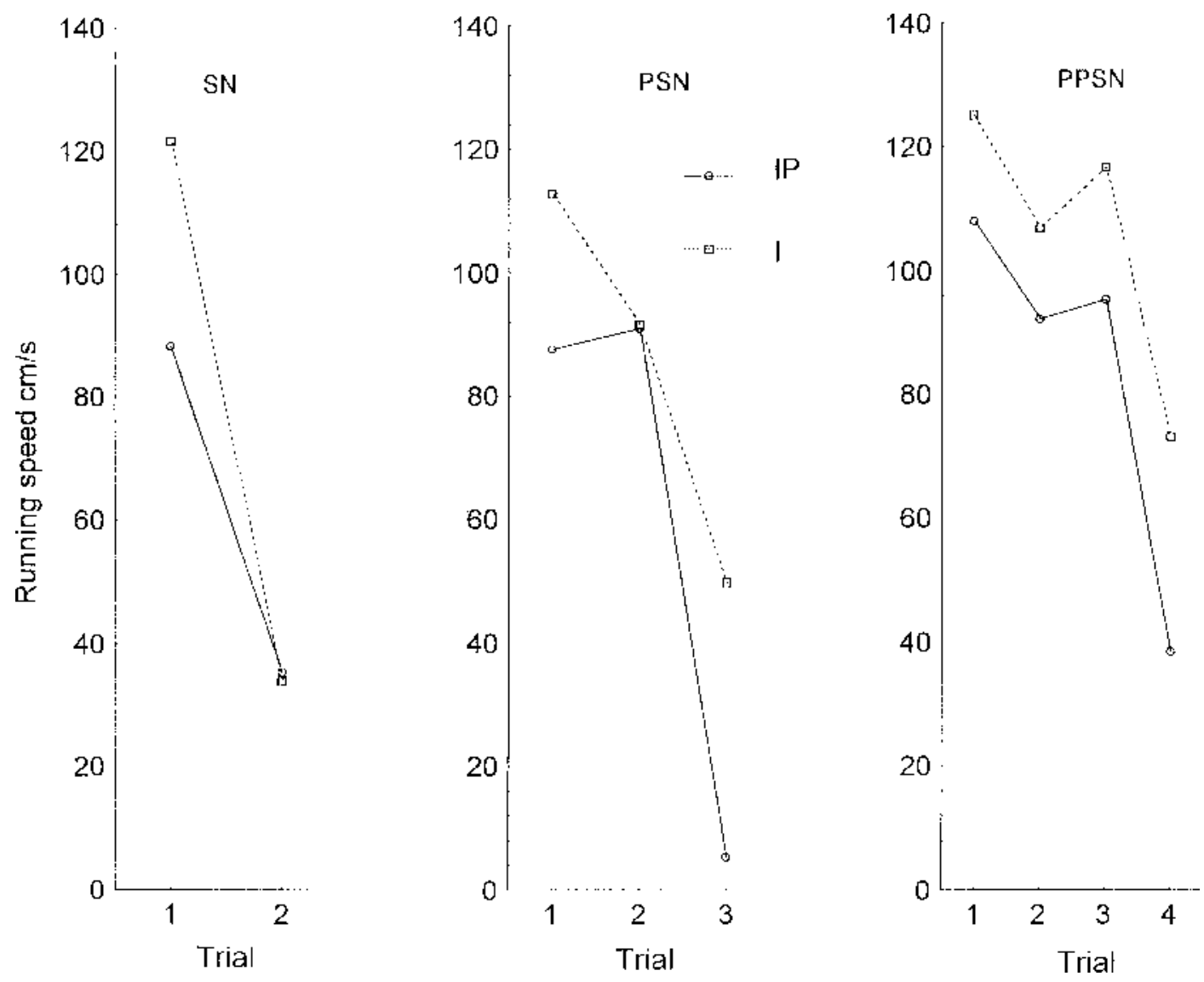

Figure 6. Running speed for Groups IP and I on each of the series received in Phase 4: the SN series (left panel), the PSN series (center panel), and the PPSN series (right panel).

first occasion it received the $\mathrm{SN}$ series indicates that item cues acquired a strong tendency to signal items under PSN training, strong enough apparently to completely oppose the also strong tendency of Trial 2 position cues to elicit fast running. In Phase 4, Group IP ran rapidly on Trial 3 of the PPSN series, a position that was associated with nonreward and slow running prior to shift, supplying additional evidence that under the PSN series, item cues were employed to signal items.

\section{GENERAL DISCUSSION}

The shift findings obtained in Experiments 1 and 2 support two major conclusions. First, the rats trained in such a way that both position and item cues validly signaled items XNY and ZNN in a black and white runway, respectively, in Experiment 1, and PSN in a gray runway in Experiment 2, employed item memories signaled items. Second, interevent anticipation was controlled by item memories when XNY and ZNN occurred both in different runways (black and white) and in the same runway (gray). The present findings complement shift findings, indicating that under $\mathrm{XNY}$ and $\mathrm{ZNN}$ in different run- ways (Burns et al., 1999) and under PSN training in the same runway (Burns et al., 2000), position cues were employed to signal items. Taking the two sets of shift findings together in combination with those supplied by Capaldi and Miller (1988) and Capaldi et al. (1997), it appears that under either the XNY and ZNN series in different runways or the PSN series in the same runway, rats employ a stimulus compound consisting of both item memories and position cues to signal items. To our knowledge, there are no other findings that so strongly support such a conclusion. That is, although some previous findings support item learning (see, e.g., Capaldi, 1994) and others position learning (e.g., Chen, Swartz, \& Terrace, 1997), none indicates so clearly that item cues and position cues may work together to signal items. There has been a tendency in investigations that have supplied evidence for either position cues or item cues to suggest, either directly or indirectly, that such evidence is evidence against control by the other sort of cue (see, e.g., Slamecka, 1964, 1985). That conclusion, whether direct or implied, is not warranted logically nor, given the findings cited above, is it warranted on the basis of experimental evidence. 
The findings cited above indicate that, when both item cues and position cues are valid signals for items, as they often are in serial learning, rats are capable of employing both together as signals for items. However, the generality of the present findings remains to be determined. That is, it may be premature to conclude that, because rats are capable of employing both sorts of cues to anticipate items under the present experimental conditions, they will necessarily do so under most other experimental conditions. In any event, the fact that rats are capable of employing both item cues and position cues together to signal items raises the possibility that mutual overshadowing occurs between them. There is as yet no direct evidence that item cues and position cues overshadow each other in serial tasks. However, since the time Capaldi et al. (1984) first suggested that, in serial learning situations, item cues may be overshadowed by various exteroceptive cues, evidence supporting that particular hypothesis has been obtained (Capaldi, Birmingham, \& Miller, 1999; see also Stempowski, Carman, \& Fountain, 1999). Given these subsequent findings, there would not seem to be any good reason to doubt that item cues and position cues may overshadow each other.

The fact that Group IP performed about as well as Group I on the SN and PPSN series in Phase 4 of Experiment 2 could be taken to suggest that little or no overshadowing of item cues by position cues occurred in Group IP. An alternative possibility is that overshadowing between item cues and position cues occurred initially in Group IP but was eventually overcome or reduced by the extensive training received by Group IP prior to Phase 4, consistent with results supplied by Bellingham and Gillette (1981). Another possibility is that item cues were weaker signals for items in Group IP than in Group I but that this difference was not reflected in serial responding, as measured by running speed. If so, assessing item learning under PSN training employing some other dependent variable would be in order.

The extent to which item cues and position cues may overshadow each other depends, no doubt, on a variety of factors. We have already indicated that such overshadowing may be reduced or eliminated by extensive training (see, e.g., Bellingham \& Gillette, 1981). Too, it appears possible, if not likely, that, relative to item cues, position cues may gain more control over responding when the XNY and ZNN series occur in different runways rather than in a single runway. Compiling a potential list of such factors would at this time be speculative. Recognizing that overshadowing and, by implication, blocking may play a role in serial learning has an important theoretical consequence: It tends to emphasize that the processes that govern serial learning are similar, if not identical, to those involved in other forms of instrumental learning. This position appears to be somewhat controversial: Some consider serial learning to involve concept learning and thus to be rather more complex than many other varieties of instrumental learning (see, e.g., Domjan, 1998; Stempowski et al., 1999). Overshadow- ing and blocking aside, Capaldi and his co-workers (e.g., Capaldi et al., 1984) have consistently suggested that serial learning is a form of discrimination learning that can be explained in the same terms as other more conventional forms of discrimination learning. In this connection, it should be noted that the item explanation applied to serial learning has been applied to a considerable variety of other instrumental learning phenomena (see, e.g., Capaldi, 1967, 1994). For example, under an irregular schedule of partial reward, animals may, with training, come to learn various relationships built into the schedule, such as that reward is highly probably following, say, four nonrewarded trials in a row. According to this view, as is perhaps obvious, explicit serial learning situations have a great deal in common with other forms of instrumental learning since item learning is a major factor in both sorts of situations.

The present results, along with a variety of other serial learning and more conventional instrumental learning data (see, e.g., Capaldi, 1994; Capaldi \& Birmingham, 1997), indicate that reward expectancy on a trial is regulated by the reward schedule associated with the item memory retrieval on that trial. For example, judging by running speed on Trial 2 of the ZNN series, expectancy of reward was very low when Item $\mathrm{Z}$ was remembered. Conventional views tend to suggest that expectancy is regulated by the reward schedule associated with various exteroceptive cues (e.g., apparatus cues). It is important to recognize that often the reward schedule associated with some item memory and that associated with, say, apparatus cues may be seriously discrepant (see, e.g., Capaldi, 1994; Capaldi et al., 1999). If results of the sort provided here are any indication, a proper understanding of expectancy requires that we attend to the reward schedules associated with both item memories and exteroceptive cues, reward schedules that are often seriously discrepant.

\section{REFERENCES}

Bellingham, W. P., \& Gillette, K. (1981). Attenuation of overshadowing as a function of nondifferential compound conditioning trials. Bulletin of the Psychonomic Society, 18, 218-220.

Burns, R. A., Dunkman, J. A., JR., \& Detloff, S. L. (1999). Ordinal position in the serial learning of rats. Animal Learning \& Behavior, 27, 272-279.

Burns, R. A., Kinney, B. A., \& Criddle, C. R. (2000). Position cues and reward memories as compatible components of serial learning. Learning \& Motivation, 31, 236-250.

CAPALDI, E. J. (1967). A sequential hypothesis of instrumental learning. In K. W. Spence \& J. T. Spence (Eds.), Psychology of learning and motivation (Vol. 1, pp. 67-156). New York: Academic Press.

CAPAldi, E. J. (1992). Levels of organized behavior in rats. In W. K. Honig \& G. Fetterman (Eds.), Cognitive aspects of stimulus control (pp. 385-404). Hillsdale, NJ: Erlbaum.

CAPALDI, E. J. (1994). The sequential view: From rapidly fading stimulus traces to the organization of memory and the abstract concept of number. Psychonomic Bulletin \& Review, 1, 156-181.

Capaldi, E. J., Alptekin, S., Miller, D. J., \& Birmingham, K. M. (1997). Is discriminative responding in reward outcomes serial learning mediated by item memories or by position cues? Learning \& Motivation, 28, 153-169.

Capaldi, E. J., \& Birmingham, K. M. (1998). Reward produced mem- 
ories regulate memory-discrimination learning, extinction, and other forms of discrimination learning. Journal of Experimental Psychology: Animal Behavioral Processes, 24, 254-264.

Capaldi, E. J., Birmingham, K. M., \& Miller, R. M. (1999). Forming chunks in instrumental learning: The role of overshadowing. Animal Learning \& Behavior, 27, 221-224.

CAPAldi, E. J., \& Miller, D. J. (1988). The rat's simultaneous anticipation of remote events and current events can be sustained by event memories alone. Animal Learning \& Behavior, 16, 1-7.

Capaldi, E. J., Verry, D. R, Nawrocki, T. M., \& Miller, D. J. (1984). Serial learning, interitem associations, phrasing cues, interference, overshadowing, chunking, memory, and extinction. Animal Learning \& Behavior, 12, 7-20.

Chen, S., Swartz, K., \& Terrace, H. S. (1997). Knowledge of the ordinal position of list items in Rhesus monkeys. Psychological Sciences, $\mathbf{8}, 80-86$.

Domjan, M. (1998). The principles of learning and behavior. Pacific Grove, CA: Brooks/Cole.

NEATH, I., \& CAPALDI, E. J. (1996). A "random-walk" simulation model for multiple-pattern learning in a radial-arm maze. Animal Learning \& Behavior, 24, 206-210.
Slamecka, N. J. (1964). An inquiry into the doctrine of remote associations. Psychological Review, 71, 61-76.

SLAMECKA, N. J. (1985). Ebbinghaus: Some associations. Journal of Experimental Psychology: Learning, Memory, \& Cognition, 11, 414-435.

Stempowski, N. K., Carman, H. M., \& Fountain, S. B. (1999). Temporal phrasing and overshadowing in rat serial-pattern learning. Learning \& Motivation, 30, 74-100.

Woodworth, R. S. (1938). Experimental psychology. New York: Holt.

\section{NOTE}

1. Under PPN, PSN, SSN, and SPN training, the terminal $\mathrm{N}$ item can be anticipated by employing the memory of the previous two items in the list - that is, $\mathrm{P}+\mathrm{P} \rightarrow \mathrm{N}$, and so forth. Employing the memory of two items as a signal for $\mathrm{N}$ is expected to require more training than employing the memory of a single prior item as a signal for $\mathrm{N}$ as in $\mathrm{SN}$, PSN, and PPSN training (see, e.g., Capaldi, 1994; Capaldi et al., 1997).

(Manuscript received April 6, 2000; revision accepted for publication October 2, 2000.) 